\title{
RACK-1 Directs Dynactin-dependent RAB-11 Endosomal Recycling during Mitosis in Caenorhabditis elegans
}

\author{
Erkang Ai, Daniel S. Poole, and Ahna R. Skop \\ Laboratory of Genetics, University of Wisconsin-Madison, Madison, WI 53706
}

Submitted September 9, 2008; Revised December 14, 2008; Accepted January 8, 2009

Monitoring Editor: Yu-Li Wang

\begin{abstract}
Membrane trafficking pathways are necessary for the addition and removal of membrane during cytokinesis. In animal cells, recycling endosomes act as a major source of the additional membranes during furrow progression and abscission. However, the mechanisms and factors that regulate recycling endosomes during the cell cycle remain poorly understood. Here, we show that the Caenorhabditis elegans Receptor of Activated C Kinase 1 (RACK-1) is required for cytokinesis, germline membrane organization, and the recruitment of RAB-11-labeled recycling endosomes to the pericentrosomal region and spindle. RACK-1 is also required for proper chromosome separation and astral microtubule length. RACK-1 localizes to the centrosomes, kinetochores, the midbody, and nuclear envelopes during the cell cycle. We found that RACK-1 directly binds to DNC-2, the C. elegans p50/dynamitin subunit of the dynactin complex. Last, RACK-1 may facilitate the sequestration of recycling endosomes by targeting DNC-2 to centrosomes and the spindle. Our findings suggest a mechanism by which RACK-1 directs the dynactin-dependent redistribution of recycling endosomes during the cell cycle, thus ensuring proper membrane trafficking events during cytokinesis.
\end{abstract}

\section{INTRODUCTION}

Membrane trafficking and remodeling pathways coordinate the delivery and dynamics of new membrane during cytokinesis (Shuster and Burgess, 2002; Danilchik et al., 2003; Albertson et al., 2005; Glotzer, 2005). As the cleavage furrow ingresses, lipids and transmembrane proteins are targeted to the ingressing furrow (Emoto and Umeda, 2000; Thompson et al., 2002). During the final phase of cytokinesis, abscission, elaborate membrane targeting, fusion, and retrieval mechanisms rely on the microtubules and actin cytoskeleton that allow for daughter cell separation (Skop et al., 2001; Gromley et al., 2005; Kouranti et al., 2006; Li et al., 2007). Many studies show that Golgi-derived vesicles are a key source of the membrane addition to the furrow (Lecuit and Wieschaus, 2000; Sisson et al., 2000; Skop et al., 2001). Work from the Toomre laboratory has shown that Golgi-derived vesicles are targeted to the furrow and midbody regions from both daughter cells (Goss and Toomre, 2008).

Another important source of new membrane during furrow formation and completion is from endocytic membrane trafficking events, in particular from the recycling endosome (RE) (Skop et al., 2001; Hickson et al., 2003; Fielding et al., 2005). During mitosis, REs localize to the pericentriolar region, in which they sort and target vesicles to the plasma membrane (Maxfield and McGraw, 2004). Proper RE function requires activity of the small GTPase Rab11 (Ullrich et al., 1996; Hickson et al., 2003; Pelissier et al., 2003; Fielding et al., 2005) and its binding partner Nuf/FIP3 (Rothwell et al., 1999; Hickson et al., 2003; Prekeris, 2003; Riggs et al., 2003). Dis-

This article was published online ahead of print in $M B C$ in Press (http://www.molbiolcell.org/cgi/doi/10.1091/mbc.E08-09-0917) on January 21, 2009.

Address correspondence to: Ahna R. Skop (skop@wisc.edu).

Abbreviations used: RE, recycling endosome; DIC, differential interference contrast; WT, wild type. ruption of either protein leads to defects in actin recruitment to the contractile ring and membrane delivery to the ingressing furrow, which lead to cytokinesis failures (Rothwell et al., 1998, 1999; Skop et al., 2001; Wilson et al., 2005). In mammalian cells, Rab11 and the Nuf homologue FIP3 interact with the exocyst complex and are necessary for delivery of new membrane to the tip of the furrow and the abscission site (Fielding et al., 2005; Wilson et al., 2005). However, questions remain as to how recycling endosomes are targeted and regulated during the cell cycle.

Our study identified an adapter protein, RACK-1, as a significant factor required for proper RE distribution during mitosis. Receptor of Activated C Kinase 1 (RACK1) was identified in a proteomic screen of isolated mammalian midbodies. Knockdown of the C. elegans homologue, K04D7.1/ RACK-1, resulted in cytokinesis failures in the early C. elegans embryo (Skop et al., 2004). The RACK1 homologue in Trypanosoma brucei, TRACK, has also been shown to be required for cytokinesis and cell growth (Rothberg et al., 2006), suggesting that the role of RACK1 in cytokinesis is likely conserved. Structurally, RACK1 consists of seven WD40 repeats forming a seven-bladed $\beta$-propeller (Ron et al., 1994), which interacts with diverse proteins with distinct structural folds (Neer et al., 1994) and has homology to the $\beta$-subunit of heterotrimeric $G$ proteins (Dell et al., 2002). RACK1 has been proposed to anchor numerous proteins at various cellular locations, including the plasma membrane (Mochly-Rosen and Kauvar, 1998), cytoskeleton (Won et al., 2001), and the $40 S$ ribosome (Nilsson et al., 2004). Despite all of the work on RACK1 in the past few years, the exact function of RACK1 during the cell cycle is unclear.

In this work, we characterized the role of C. elegans RACK-1 in multiple processes during mitosis in one-cell embryos. We also show that RACK-1 interacts with the p50/dynamitin subunit of the dynactin complex and suggest that RACK-1 regulates the distribution of the RE by targeting the dynactin complex to the centrosomes. 


\section{MATERIALS AND METHODS}

\section{Worm Strains}

Strains used include N2 (wild type); OD58 (GFP-PH ${ }^{\mathrm{PLC} 181}$ ) (Audhya et al., 2005); WH258 (GFP-DNC-2) (Zhang et al., 2008a); WH347 (GFP-RAB-11) (Zhang et al., 2008b); WH204 (GFP-TBB-2) (Strome et al., 2001); AZ212 (GFP-H2B) (Praitis et al., 2001); MG170 (ZEN-4-GFP) (Kaitna et al., 2000). N2 worms were cultured at $20^{\circ} \mathrm{C}$ as described by Brenner (1974). All green fluorescent protein (GFP) strains were maintained at $25^{\circ} \mathrm{C}$.

\section{RNA Interference (RNAi)}

RNAi of rack-1 was performed by the feeding method (Timmons et al., 2001). Larval stage 4 (L4) hermaphrodites were fed on bacteria expressing doublestranded RNA for $30-48 \mathrm{~h}$ at $25^{\circ} \mathrm{C}$ or $48-60 \mathrm{~h}$ at $20^{\circ} \mathrm{C}$ before analysis. rackand $z g y-9$ RNAi feeding bacteria were obtained from the Ahringer RNA library (Kamath and Ahringer, 2003), and sequence were verified. rab-11 RNAi feeding vector was a gift from John White's laboratory (Zhang et al. $2008 \mathrm{~b}$ ) and was transformed into HT115 bacteria. $d n c-2$ RNAi feeding bacteria was created by cloning $d n c-2$ cDNA into the pL4440 plasmid and then transforming the construct into HT115 bacteria.

\section{Western Blotting}

Total SDS-soluble protein was extracted from 90 rack-1(RNAi) or control worms (worms grown on bacteria containing empty pL4440 vector). The extracts were separated on a 10\% SDS-polyacrylamide gel electrophoresis (PAGE) gel, and then blotted and probed with a 1:1000 dilution of antiRACK1 antibody (catalog no. sc-10775; Santa Cruz Biotechnology, Santa Cruz, CA) and a 1:4000 dilution of anti-actin antibody (catalog no. 69100; MP Biomedicals, Irvine, CA) as a control. Blot signals were detected using a horseradish peroxidase-conjugated secondary antibody (1:3000; catalog no. 170-6516 [mouse] and 170-6515 [rabbit]; Bio-Rad, Hercules, CA) and Opti4CN substrate (catalog no. 170-8235; Bio-Rad).

\section{Immunostaining}

Immunostaining was performed as described previously (Skop and White 1998). rack-1(RNAi) and wild-type embryos were stained with anti-RACK1 (1:100, catalog no. sc-10775; Santa Cruz Biotechnology), anti-RAB-11 (obtained from Spang laboratory; Poteryaev et al., 2007), anti-PLK-1 (obtained from the Golden laboratory; Chase et al., 2000), and anti-tubulin (catalog no. 69125; MP Biomedicals) antibodies. The secondary antibodies used in this study were anti-rabbit Alexa Fluor-488 (catalog no. A11008; Invitrogen, Carlsbad, CA) and anti-mouse Alexa Fluor-568 (catalog no. A11004; Invitrogen). DNA was counterstained with TOTO-3 at a 1:4000 dilution (catalog no. T3604; Invitrogen). Embryos were mounted in $8 \mu \mathrm{l}$ of VECTASHIELD (catalog no. H-1200; Vector Laboratories, Burlingame, CA) and examined using an LSM510 confocal microscope with an A-Plan Apochromat 100×/1.46 objective (both from Carl Zeiss, Jena, Germany).

\section{Time-Lapse Microscopy}

Embryos were dissected in $15 \mu \mathrm{l}$ of blastomere culture media (Skop et al. 2001 ) on $22-\times 22-m m$ coverslips coated with poly-L-lysine (catalog no. P1524 Sigma-Aldrich, St. Louis, MO) with vacuum grease feet. Slides were placed on top of the coverslips and sealed with silicon oil. Time-lapse videos were recorded at 10-s intervals by using a 200M inverted Axioscope microscope (Carl Zeiss) equipped with a spinning disk confocal scan head (QLC100; VisiTech, Sunderland, United Kingdom), an Orca ER charge-coupled device camera (Hamamatsu) and a differential interference contrast (DIC) camera (Hamamatsu). Images were acquired using a $63 \times, 1.4$ NA Plan-Apochromat objective and Openlab software (Improvision, Coventry, United Kingdom). For GFP-DNC-2 movies, embryos were mounted in blastomere culture media on $2 \%$ agarose pads. Four Z-series images were acquired at $0.5-\mu \mathrm{m}$ step every $20 \mathrm{~s}$. The images were then projected using ImageJ and Image5D (Abramoff et al., 2004).

\section{Quantification}

To measure the length of the astral microtubules (see Figure $2 \mathrm{Q}$ ), the five longest microtubules from eight wild-type and eight rack-1(RNAi) embryos at metaphase or anaphase were measured in Openlab, and the average length is plotted. To measure the intensities of RAB-11 around the centrosomes (see Figure $4 \mathrm{M}$ ), five wild-type, five rack-1(RNAi), and five $d n c-2(R N A i)$ metaphase embryos stained with RAB-11 antibody were selected and a circular region with a 9- or 8- $\mu \mathrm{m}$ diameter was drawn around anterior or posterior asters, respectively. Intensities measured using Openlab software (Improvision) were averaged and normalized to wild type. In all of the quantifications, two-tailed Student's $t$ test was used.

\section{Immunoprecipitation}

Adult hermaphrodites (GFP-DNC-2 or N2) suspended in homogenization buffer (50 mM HEPES, pH 7.6, $140 \mathrm{mM} \mathrm{KCl}, 1 \mathrm{mM}$ EDTA, 10\% glycerol, $5 \mathrm{mM}$ dithiothreitol, $0.5 \%$ NP-40, $\%$ phenylmethylsulfonyl fluoride [PMSF], and protease inhibitor cocktail [Research Products International, Mt. Prospect, IL] were lysed by sonication. Lysates were centrifuged at $4^{\circ} \mathrm{C}$ at $20,000 \mathrm{rpm}$ for 20 min. Protein A-agarose beads were incubated with GFP antibody (catalog no. 632381; Clontech, Mountain View, CA) in immunoprecipitation (IP) buffer (50 mM HEPES, pH 7.6, $140 \mathrm{mM} \mathrm{KCl}, 50 \mathrm{mM}$ EDTA, 0.05\% NP-40, 2\% PMSF, and protease inhibitor cocktail) for $4 \mathrm{~h}$ at $4^{\circ} \mathrm{C}$. Beads were washed and incubated with precleared lysates at $4^{\circ} \mathrm{C}$ overnight and washed three times with ice-cold IP buffer. Precipitates were boiled in sample buffer and run on a $10 \%$ SDSPAGE gel. Western blots were performed to detect RACK-1 by using antiRACK1 antibody (catalog no. sc-10775; Santa Cruz Biotechnology) and an ECL kit (GE Healthcare, Little Chalfont, Buckinghamshire, United Kingdom).

\section{RESULTS}

\section{C. elegans RACK-1 Is a Homologue of Mammalian RACK1}

C. elegans RACK-1/K04D7.1 was identified in an RNAi screen of isolated mammalian midbody protein homologues (Skop et al., 2004). RACK-1 is a homologue of mammalian RACK1. Sequence analysis revealed that RACK-1 shares $73 \%$ identity and $97 \%$ similarity at the amino acid level with mammalian RACK1. RACK-1 is also highly conserved among eukaryotes from yeast to humans. Structurally, both C. elegans RACK-1 and human RACK1 belong to a large family of WD40 repeat proteins that share homology to the $\beta$ subunit of heterotrimeric $G$ proteins (Dell et al., 2002). The WD40 repeats adopt a circular seven-bladed $\beta$-propeller structure (Ron et al., 1994). RACK1 has been proposed to anchor proteins at particular cellular loci thereby facilitating the formation of specific signaling complexes (McCahill et al., 2002; Sklan et al., 2006).

\section{RACK-1 Is Required for Cytokinesis}

We used RNAi by feeding to test for the requirement of RACK-1 in the early embryo. RACK-1 protein levels were assessed at different time points by Western blot analysis. RACK-1 was knocked down after $24 \mathrm{~h}$ at 20 or $25^{\circ} \mathrm{C}$. After $48 \mathrm{~h}$ of RNAi feeding at $20^{\circ} \mathrm{C}$ or $25^{\circ} \mathrm{C}$, the RACK- 1 protein was not detectable (Supplemental Figure S1A). RNAi of RACK-1 led to $48 \%$ embryonic lethality after $48-72 \mathrm{~h}$ feeding at $20^{\circ} \mathrm{C}$ and $54 \%$ embryonic lethality after $24-48 \mathrm{~h}$ feeding at $25^{\circ} \mathrm{C}$. A reduction in the brood size by $60 \%$ compared with wild type was also observed. Furthermore, animals grown on RNAi feeding bacteria for longer than $48 \mathrm{~h}$ at $25^{\circ} \mathrm{C}$ became sterile ( $9 / 10$ worms), suggesting defects in germline membrane formation or organization (see next section).

We used DIC microscopy to record the first two rounds of divisions in C. elegans embryos (Figure 1, A-O; Supplemental Movies 1-3). N2 worms were grown on rack-1 RNAi feeding bacteria for $42-54 \mathrm{~h}$ at $20^{\circ} \mathrm{C}$ or $36-48 \mathrm{~h}$ at $25^{\circ} \mathrm{C}$ before imaging. We noticed that rack-1(RNAi) embryos were osmotically sensitive in M9 or egg buffer. Therefore, we dissected and mounted the rack-1(RNAi) embryos by using the hanging drop method in blastomere culture medium (Skop et al., 2001), which rescued the osmotic defects. In rack-1(RNAi) embryos, polar body extrusion failures were observed (Figure 1K, 19/35 embryos), indicating failures in cytokinesis during meiosis. After meiosis the cell cycle proceeded slowly ( $\sim 7 \mathrm{~min}$ slower) from centration through rotation of the pronuclei (prometaphase), yet proceeded normally from nuclear envelope breakdown through division (late prometaphase-cytokinesis). Cell cycle progression defects have also been observed in cells depleted of TRACK, the T. brucei RACK-1 homologue (Rothberg et al., 2006). In rack-1(RNAi) embryos, cytokinesis failed in 18 of 35 embryos (51\%). In $8 / 18$ embryos, the cleavage furrow initiated but ingressed $<1 / 4$ of the cell diameter and then retracted (early cytokinesis defect, Figure 1, F-J, and Supplemental Movie 2). 
Figure 1. RACK-1 is required for cytokinesis. (A-O) Selected DIC images from time-lapse sequences of one wild-type and two rack-1(RNAi) embryos are shown. (A-E) A wild-type embryo from pronuclei migration until the end of cytokinesis, also see Supplemental Movie 1. Cleavage furrow ingression initiates during anaphase (C) and completes during telophase (D), partitioning the nuclei to two daughter cells (E). (F-J) A RACK-1 depleted embryo exhibiting early cytokinesis defect; also see Supplemental Movie 2. The cleavage furrow initiates and ingresses $<1 / 4$ of the cell diameter (I) and then regresses $(\mathrm{J})$. (K-O) A RACK-1-depleted embryo exhibiting late cytokinesis defect; also see Supplemental Movie 3. The cleavage furrow initiates and ingresses to apparent completion $(\mathrm{N})$, but regresses soon after $(\mathrm{O})$. (P-W) RACK-1 localization during the cell cycle. Top (P-S), embryos stained with mammalian RACK1 antibody. Bottom (T-W), the same embryos counterstained with TOTO-3 to visualize the DNA. Signals of RACK-1 were detected in the cytoplasm (P-S), at spindle poles (P-R), kinetochores (Q), nuclear envelopes (P and S), and the midbody

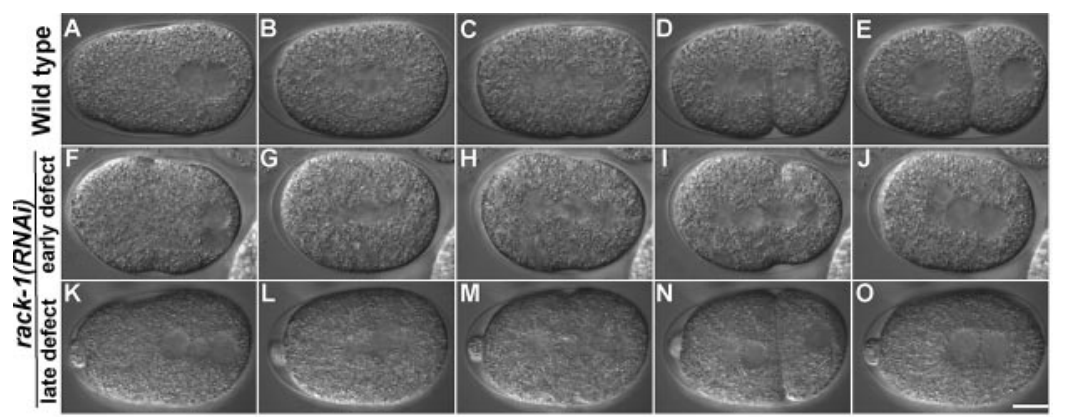
(S). (X) rack-1 RNAi causes germline cytokinesis defects. Gonads from wild-type and rack-1(RNAi) worms expressing GFP-PH ${ }^{\mathrm{PLC} 1 \delta 1}$ are used to visualize the plasma membrane. At top focal plane, wild-type germline displays equal-sized membranes with hexagonal shape. In the rack-1(RNAi) germline, the cell size varies and the plasma membranes are sometimes missing. At midfocal plane, the T-shape membranes seen in the wild-type germline are now replaced with Y-shape, I-shape, and loop-shape membranes in the rack-1(RNAi) germline (arrows). Bars, $10 \mu \mathrm{m}$.

In the other 10/18 embryos that failed cytokinesis, the cleavage furrow fully ingressed but failed to complete cytokinesis and the cleavage furrow regressed (late cytokinesis defect, Figure 1, K-O, and Supplemental Movie 3). Our observations suggest that RACK-1 is necessary for both furrow ingression and completion/abscission during cytokinesis. These data are consistent with results from T. brucei that when TRACK was depleted, the bloodstream forms displayed a delay in the onset of cytokinesis, whereas the procyclic forms arrested midway through cell cleavage (Rothberg et al., 2006). Last, we observed other defects in rack-1(RNAi) embryos that failed cytokinesis. Some embryos were rounder and smaller than wild type (5/18 embryos), and some embryos had areas depleted of yolk granules (Figure 1, M and N, 8/18 embryos). These additional defects likely reflect the multiple roles RACK-1 may play during growth and development.

\section{RACK-1 Is Necessary for Germline Membrane Organization}

To address whether the sterility we observed was due to germline defects, a GFP-PH ${ }^{\mathrm{PLC} 1 \partial 1}$ strain (Audhya et al., 2005) was used to visualize germline plasma membrane organization. In wild-type hermaphrodite animals, the membranes in the gonad arm were arranged in equal-sized hexagonal shapes at the top focal plane (Figure 1X). At the midfocal plane, T-shaped membranes line up at the periphery surrounding the rachis. In the germline of rack-1(RNAi) worms, membranes formed random shapes and were sometimes discontinuous. At the midfocal plane, T-shapes were replaced with loops, Y-shapes, or I-shapes (arrows, Figure 1X, bottom right), suggesting abnormalities in membrane remodeling or formation during germline development. RACK-1 has a punctate localization pattern in the germline but did not seem to localize to any specific structures (Supplemental Figure S1, H and I).

The membrane structure in the C. elegans gonad resembles the polarized epithelium during cellularization in the Drosophila embryo, in which furrows invaginate from the cortex and separate the nuclei (Lecuit and Wieschaus, 2000). The germline phenotype we observe in RACK-1-depleted worms was reminiscent of rab11 and nuf mutants in the Drosophila embryo (Rothwell et al., 1999). When Rab11 and Nuf are absent, actin-labeled hexagonal cortices are disrupted and discontinuous (Rothwell et al., 1999). In addition, similar phenotypes have been observed in the germlines of RAB-11-depleted animals in C. elegans (Skop et al., 2001). The small GTPase Rab11 and its effecter Nuf reside at the recycling endosomes and stimulate the delivery of RE-derived vesicles to the site of the cleavage furrow (Hickson et al., 2003; Wilson et al., 2005). The similarities between the phenotypes suggest a possible relationship in function between RACK-1 and RE component proteins.

\section{RACK-1 Localization in C. elegans Embryos}

To determine the subcellular localization of RACK-1 during the cell cycle, a polyclonal RACK1 antibody raised against human RACK1 (Santa Cruz Biotechnology) was used to immunostain C. elegans embryos. To verify the specificity of the antibody, we depleted RACK-1 by feeding RNAi and immunostained these embryos. In rack-1(RNAi) embryos, RACK-1 signals were greatly weakened in the cytoplasm and were lost from specific loci, indicating the antibody used is specific to RACK-1 in C. elegans (Supplemental Figure S1, B-D). As a control, RNAi of RACK-1 did not affect PLK-1 localization (Supplemental Figure S1, E-G), which shared a similar staining pattern (Chase et al., 2000) with RACK-1. The specificity of the antibody was also confirmed by Western blot, in which the antibody detected a single band with the predicted size ( $36 \mathrm{kDa}$, Supplemental Figure S1A), and it was absent after RACK-1 depletion by RNAi (Supplemental Figure S1A). Throughout the cell cycle, RACK-1 was dispersed in the cytoplasm in small puncta (Supplemental Figure 1, P-S), which may be ribosomes (Nilsson et al., 2004). At metaphase, RACK-1 predominantly localized to the centrosomes and also accumulated at kinetochores (Figure 1Q). As the centrosomes and chromosomes separate in anaphase, RACK-1 protein levels were no longer robust at 

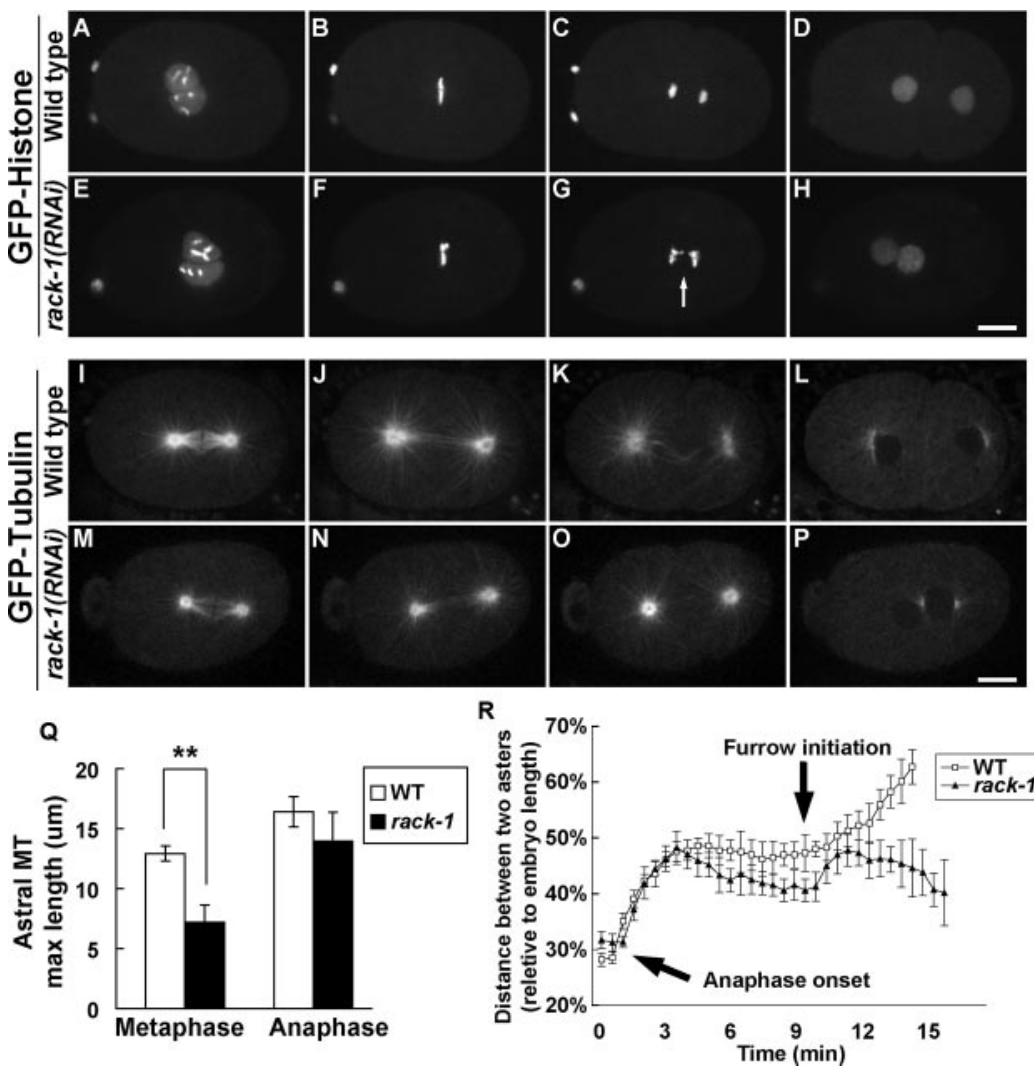

Figure 2. RACK-1 depletion affects chromosome segregation and astral microtubule length. (A-H) Time-lapse sequences are from embryos expressing GFP-H2B. (A-D) In wild-type embryos, chromosome congression (B) and separation (C) are observed. $(\mathrm{E}-\mathrm{H})$ : rack-1(RNAi) embryos display abnormal nuclear shape (E) and failure in chromosome congression $(\mathrm{F})$. Lagging chromosomes $(\mathrm{G}$, arrow) are also observed. (I-P) Selected images are from time-lapse sequences of embryos expressing GFPtubulin to visualize microtubules. (I-L) In wild type (also see Supplemental Movie 4), the spindle sets up at metaphase (I), elongates during anaphase (J), and is bundled by the ingressing furrow to form the spindle midzone (K). (M-P) In the rack-1(RNAi) embryos (also see Supplemental Movie 5), faint remnants of the bundled spindle midzone are observed $(\mathrm{O})$, but soon disappear as the furrow regresses. (Q) During metaphase, the lengths of astral microtubules in rack1(RNAi) embryos are shorter than that in WT embryos (I and M). Five of the longest microtubules from each of eight wild-type and eight rack-1(RNAi) embryos were measured using Openlab software, and the average lengths are plotted. (R) The change in spindle length measured as the percentage of egg length is plotted against time using three wild-type and three rack-1(RNAi) embryos. Time is relative to metaphase chromosome congression. Bars, $10 \mu \mathrm{m}$; error bars, SD. Asterisks indicate a statistically significant difference $(\mathrm{p}<0.01)$; Student's $t$ test, two-tailed equal variance. the kinetochores (Figure 1R). RACK-1 localized to the midbody after completion of cytokinesis (Supplemental Figure 1S) and could be detected at the nuclear envelopes at this time (Supplemental Figure 1S). In Chinese hamster ovary cells, using the same antibody, RACK1 was found at centrosomes and midbodies, although RACK1 was enriched along the spindle microtubules as well (data not shown). Similarly, trypanosome RACK1 was also found in the cytoplasm and concentrated in the perinuclear region (Rothberg et al., 2006), possibly the spindle poles, suggesting that the localization and cellular function is likely conserved.

\section{RACK-1 Is Required for Proper Chromosome Separation}

Because RACK-1 localized to kinetochores, we examined chromosome and nuclear dynamics in rack-1(RNAi) embryos by using an GFP-HistoneH2B strain that visualizes the chromosomes (Praitis et al., 2001) (Figure 2, A-H). In RACK-1-depleted embryos, nuclear shape and chromosome congression was abnormal (Figure 2, E and F). Lagging chromosomes and chromosome bridges were also observed (Figure 2G, 4/12 and $2 / 12$ embryos, respectively). Meiotic chromosomes often segregated together with the embryonic pronuclei, forming bridge-like structures (3/12 embryos; data not shown). Defects in chromosome segregation, however, did not necessarily lead to cytokinesis failures, which have also been observed in other studies (O'Connell et al., 1998). The function of RACK-1 at kinetochores is unknown.

\section{RACK-1 Is Necessary for Astral Microtubule Length but Not Spindle Midzone Formation}

To determine whether microtubule dynamics were disrupted in rack-1(RNAi) embryos, we used a GFP- $\beta$-tubulin fusion strain (Strome et al., 2001) to monitor microtubule dynamics (Figure 2, I-P, and Supplemental Movies 4 and 5). In wild type, astral microtubules formed a dense radial array around the centrosomes after nuclear envelope breakdown. At metaphase, the astral microtubules were $\sim 12.9 \mu \mathrm{m}$ in length (Figure 2I). As the cell entered anaphase, astral microtubules reached the cortex and measured $\sim 16.2 \mu \mathrm{m}$ in length (Figure 2J). The two asters were pulled toward the poles and the spindle elongated. The interlacing microtubules between separating chromosomes were bundled to form the spindle midzone (Figure $2 \mathrm{~K}$ ), which was constricted by the cleavage furrow and bundled into the midbody (Figure 2L). In rack-1(RNAi) embryos, the astral microtubules were significantly shorter in metaphase $(\sim 7.2 \mu \mathrm{m}$; Figure $2 \mathrm{M}$, quantified in $2 \mathrm{Q}$ ). However, during anaphase the microtubule length became equal to that of wild type (WT); here, the microtubules reached the cortex $(\sim 14.1$ $\mu \mathrm{m}$; Figure 2, J and N). Faint remnants of the spindle midzone were observed but soon disappeared as the furrow regressed (Figure 2O). Spindle length in rack-1(RNAi) embryos was comparable with wild type during the first 3 min of anaphase but was significantly shorter $(\sim 20 \%)$ thereafter (Figure 2R).

To determine whether RACK-1 depletion affected central spindle assembly, We examined a GFP strain expressing ZEN-4 (Kaitna et al., 2000). ZEN-4 is the homologue of mammalian kinesin MKLP1, which is required for the assembly of the central spindle and the completion of cytokinesis (Mishima et al., 2002). In wild type, ZEN-4-GFP localized to microtubule bundles in the spindle midzone early in anaphase (Supplemental Figure S2B) and then to the spindle midbody (Supplemental Figure S2D). We observed a similar localization pattern in rack-1(RNAi) embryos (Supplemental 


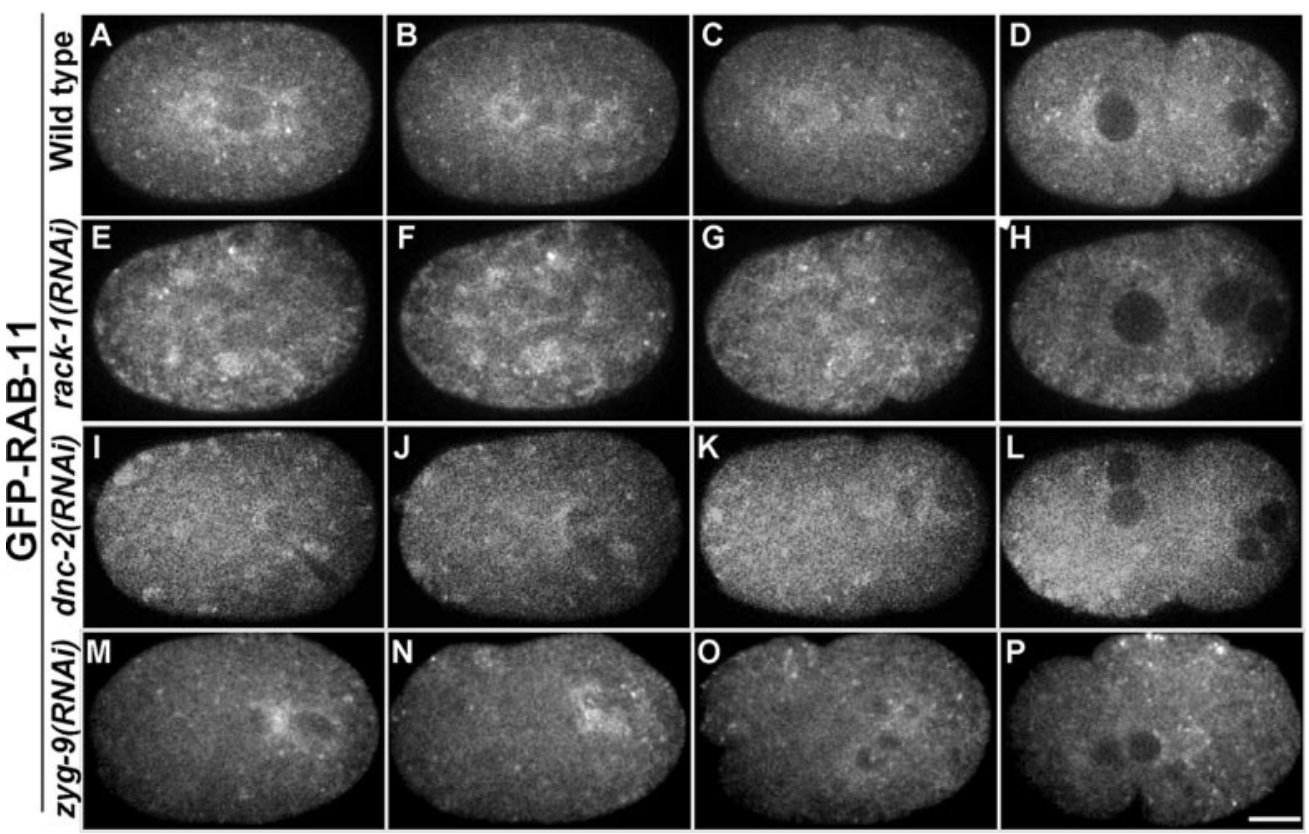

Figure 3. RACK-1 and DNC-2 are required for GFP-RAB-11 localization. Selected confocal images from time-lapse sequences of wild-type, rack-1(RNAi), dnc-2(RNAi), and zyg-9(RNAi) embryos from a GFP-RAB-11 strain are shown. (A-D) Wild-type embryo (see Supplemental Movie 6). GFP-RAB-11-labeled small vesicles are observed in the pericentriolar region during prometaphase (A). During metaphase, the pericentriolar GFP-RAB-11 is highly enriched (B). During anaphase, GFP-RAB-11 localizes along the spindle and aggregates in small cytoplasmic clumps near the spindle (C). GFP-RAB-11 localizes to the midbody (D). (E-H) rack-1(RNAi) embryo; also see Supplemental Movie 7. Pericentriolar GFP-RAB-11 signals are greatly reduced (E-G). Cytoplasmic clumps are seen starting from prometaphase and lasting through anaphase (E-G). (I-L) dnc-2(RNAi) embryo; also see Supplemental Movie 8. DNC-2 depletion causes aberrant spindle and centrosomes (I). Pericentriolar GFP-RAB-11 signals are greatly reduced (I-K). Cytoplasmic clumps are observed, particularly near the cortex (I and J). (M-P) zyg-9(RNAi) embryo. ZYG-9 depletion also causes spindle alignment defects; yet, GFP-RAB-11 signals localize to spindle poles and along the spindle (M-O). Bar, $10 \mu \mathrm{m}$.

Figure S2, F-J), except that ZEN-4-GFP disappeared when the cleavage furrow regressed (Supplemental Figure S2J). RACK-1 depletion did not affect ZEN-4 localization, indicating that RACK-1 is likely not involved in the maintenance of the central spindle.

\section{RACK-1 Is Required for RAB-11 Localization}

Because we noticed phenotypic similarities between RACK-1 and RAB-11 RNAi depletion experiments, we examined RAB-11 localization during the cell cycle by monitoring GFPRAB-11 dynamics (Zhang et al., 2008b) (Figure 3). In wildtype embryos, GFP-RAB-11-labeled vesicles localized to the pericentrosomal region as early as pronuclear centration (prophase, Figure 3A). During anaphase, GFP-RAB-11 was also found along the spindle and aggregated in small cytoplasmic clumps near the spindle (Figure 3, B and C). When RACK-1 was knocked down, GFP-RAB-11 vesicles were significantly reduced throughout the embryo and in particular at the pericentrosomal region and around the spindle (Figure 3, E-G), although some RAB-11 still localized on and around the spindle. In addition, large clumps of GFPRAB-11 were observed in the cytoplasm (Figure 3, E-H, and Supplemental Movie 7). We speculate that the clumping of GFP-RAB-11 might be due to the loss of the signal to target or localize RAB-11 to the centrosome.

To confirm the endogenous localization of RAB-11 we immunostained embryos by using a C. elegans RAB-11 antibody (Poteryaev et al., 2007). Reduced RAB-11 vesicles around the centrosomes as well as along the spindle (Figure 4, A-H) were observed, similar to what we saw using the GFP-RAB-11 strain. We quantified the RAB-11 intensity around the centrosomes and found that in rack-1(RNAi) embryos the intensity dropped $>70 \%$ compared with wild type at both anterior and posterior centrosomes (Figure 4M). However, we did not observe cytoplasmic clumping as seen in GFP-RAB-11. We speculate that the difference may be caused by the overexpression of RAB-11 in GFP-RAB-11 strain, or by disruption of membrane structure during fixation in immunostaining.

To determine whether RAB-11 is also required to target RACK-1 to centrosomes, we knocked down RAB-11 by using RNAi and immunostained for the presence of RACK-1. Despite defects in spindle alignment and cytokinesis, which have been reported previously (Skop et al., 2001), RACK-1 localized to centrosomes normally (Figure 6, D-F). The cytokinesis failures in rack-1(RNAi) embryos may be a consequence of RAB-11 mislocalization and subsequent failure to target vesicles to the cleavage site. We speculate that RACK-1 may function to recruit RAB-11 to the centrosomes, spindle, and to other cellular sites during the cell cycle.

\section{RACK-1 Regulates Recycling Endosome Distribution via the Dynactin Complex}

We next examined how RACK-1 might regulate the distribution of RAB-11. Dynein/dynactin complexes are thought to facilitate the transport and localization of endomembrane compartments in interphase cells (Valetti et al., 1999; King et al., 2003; Riggs et al., 2007). The dynein motor has been proposed to influence the localization of factors that regulate delivery of RE vesicles to the cleavage furrows (Riggs et al., 2007). Interestingly, a yeast two-hybrid screen identified DNC-2, the p50/dynamitin subunit of the dynactin com- 

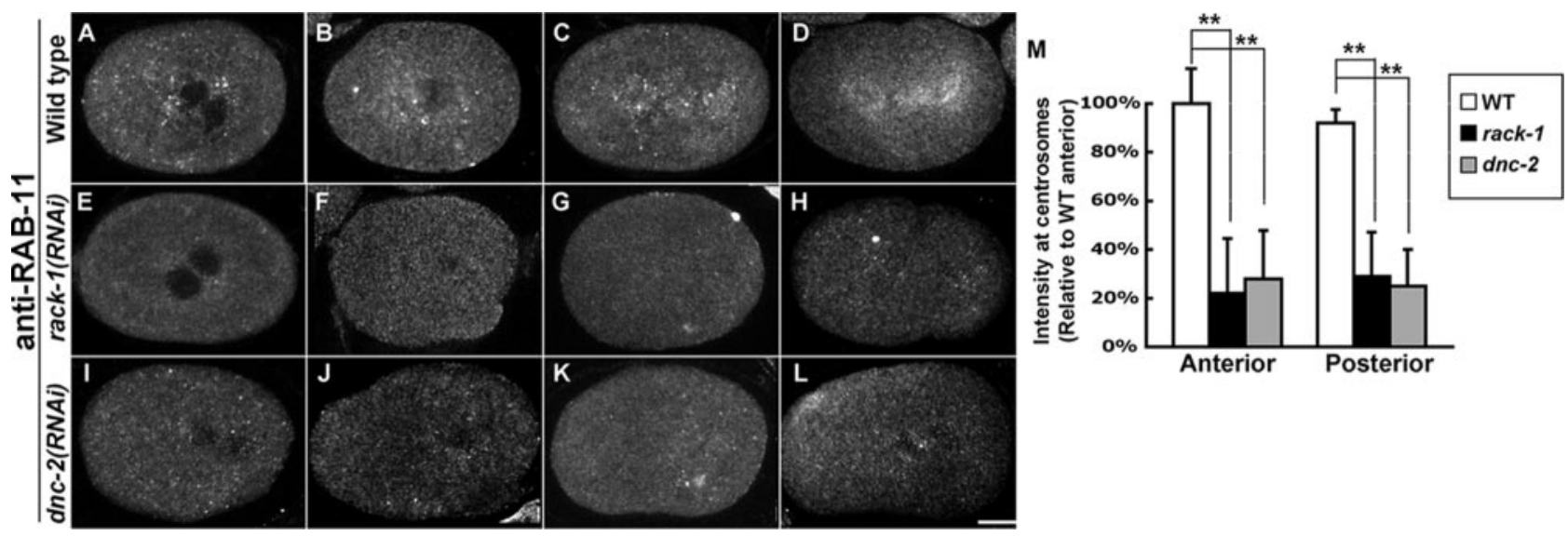

Figure 4. RACK-1 and DNC-2 are required for RAB-11 localization. Representative images are from wild-type (A-D), rack-1(RNAi) (E-H), and $d n c-2(R N A i)$ (I-L) embryos stained with anti-RAB-11 antibody. In wild type, RAB-11 concentrates around the centrosomes (A-C) and slightly along the spindle (B and C). In rack-1(RNAi) embryos, RAB-11 is greatly reduced at these regions (E-H). Similar reductions around centrosomes and the spindle are observed in $d n c-2(R N A i)$ embryos (I-L). (M) Quantification of pericentrosomal RAB-11 intensity from five wild-type, five rack-1(RNAi), and five $d n c-2(R N A i)$ embryos stained with RAB-11 antibody. Intensities of circles $9 \mu$ m in diameter around centrosomes are measured and normalized to the anterior centrosomal region in wild type Bar, $10 \mu \mathrm{m}$; error bars, SD. Asterisks indicate a statistically significant difference $(\mathrm{p}<0.01)$; Student's $t$ test, two-tailed equal variance.

plex, as a binding partner of C. elegans RACK-1 (Li et al., 2004). RAB-11-labeled recycling endosomes may be trafficked to the centrosomes and elsewhere via dynein/dynactin complexes.

To test this hypothesis, we monitored GFP-RAB-11 localization in $d n c-2(R N A i)$ embryos (Figure 3, I-L, and Supplemental Movie 8). Strikingly, depletion of DNC-2 led to GFPRAB-11 loss from the pericentriolar region and the spindle (Figure 3, I-K). We also observed a clumping of GFP-RAB-11 in the cytoplasm (Figure 3, I-K), particularly near the cortex (Figure 3I), similar to our results with RACK-1 depletion (Figure 3E). Cytokinesis defects $(1 / 18 ; 5.5 \%)$ were rarely observed in dnc-2(RNAi) embryos; however, rack-1(RNAi) embryos predominantly displayed these defects. In addition, we observed endogenous RAB-11 localization by immunostaining $d n c-2(R N A i)$ embryos with anti-RAB-11 antibody (Figure 4, I-L). In these embryos, RAB-11 vesicles were dramatically diminished around centrosomes and along the spindle throughout the cell cycle, similar to the staining pattern in rack-1(RNAi) embryos. The fluorescence intensity around the centrosomes in $d n c-2$ (RNAi) embryos was $70 \%$ less than that in wild-type embryos (Figure 4M). These results suggest that the dynein/dynactin complex is required for the transport of RE to the centrosomes and around the spindle.

Next, we tested whether RAB-11 is required for dynactin localization. We used a GFP-DNC-2 strain (Zhang et al., 2008a), which in wild type faintly decorated the centrosomes during prometaphase and then strongly localized to the spindle during metaphase and anaphase (Figure 5, A-D; and Supplemental Movie 9). In rab-11(RNAi) embryos, GFPDNC-2 properly localized to centrosomes and spindles, similar to that of wild type (Figure 5, I-L, and Supplemental Movie 11). These data indicates that RAB-11 is not necessary to target DNC-2 to the centrosomes.

Last, we determined the requirement of RACK-1 for DNC-2 localization during the early stages of mitosis. Before furrow initiation, RACK-1 may target DNC-2 to the centrosomes where RE vesicles are trafficked. When we depleted RACK-1 by RNAi, GFP-DNC-2 levels at the centrosomes and on the mitotic spindle were significantly reduced (Figure 5, E-H, and Supplemental Movie 10). Most of the GFP-
DNC-2 signal aggregated in clumps in the cytoplasm yet some remained on the spindle (Figure 5, E-F). These findings suggest that RACK-1 is in part required to target DNC-2 to the centrosomes and mitotic spindle. Spindle alignment defects, which are often seen in $d n c-2(R N A i)$ embryos (Skop and White, 1998), were not observed upon RACK-1 depletion, suggesting that DNC-2 localization to the centrosomes and the spindle is not necessary for spindle orientation. RACK-1 may regulate the distribution of dynactin by targeting it to centrosomes in particular, where RACK-1 is found (Figure 1, P-R).

Alternatively, it is also possible that RACK-1 and DNC-2 are mutually required to target each other at the centrosomes. To test this, we determined whether RACK-1 is properly localized in embryos depleted of DNC-2 (Figure 6, G-I). The majority of the embryos displayed spindle alignment defects, which were consistent with previous findings (Skop and White, 1998). In these embryos, RACK-1 still localized to centrosomes from prometaphase to anaphase (Figure 6, G-I), suggesting that DNC-2 may not be required for RACK-1 localization at the centrosomes. Together, our findings suggest that centrosomal RE targeting by RACK-1 is dynactin dependent. RACK-1 likely regulates the localization of the RE by mediating the localization of DNC-2.

\section{rack-1(RNAi) Phenotypes Are Not Due to Reduced Astral MT Length}

We observed shorter astral microtubules in rack-1-RNAitreated embryos (Figure 2Q). Is this a possible reason for the observed defects in RE and dynactin localization? For comparison, we examined the phenotypes of $z y g-9$ (RNAi) embryos, which also have shorter astral microtubules (Srayko et al., 2003). ZYG-9 is the C. elegans homologue of XMAP215 and is a centrosome component protein (Matthews et al., 1998). RNAi of ZYG-9 leads to shorter astral microtubules comparable to rack-1(RNAi) embryos (Srayko et al., 2003). Depletion of ZYG-9 by RNAi in GFP-RAB-11 and GFPDNC-2 embryos led to defects in pronuclear migration and spindle misalignment, consistent with previous findings (Matthews et al., 1998); yet, both RAB-11 and DNC-2 localization patterns were similar to wild-type embryos (Figures 3, M-P, and 5, M-P). Shorter microtubules were not ob- 

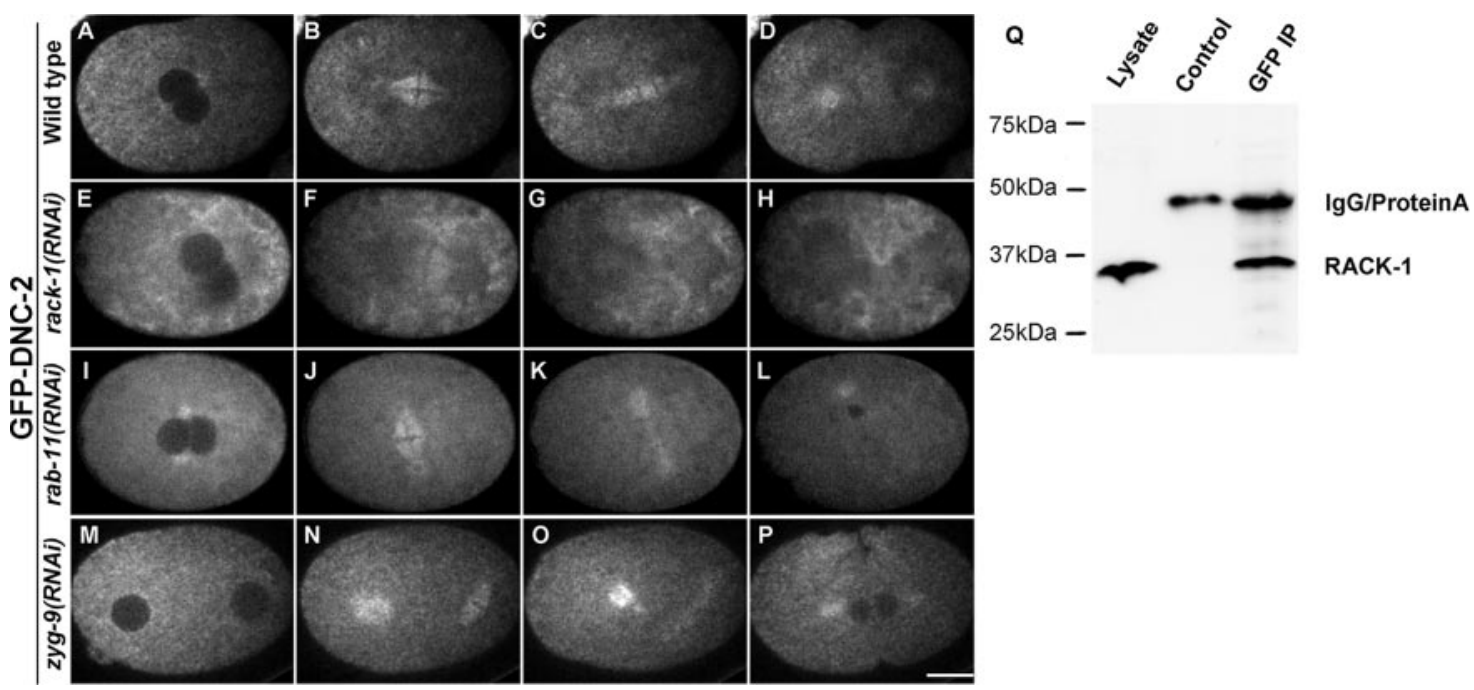

Figure 5. RACK-1 is required for DNC-2 localization. (A-P) Selected z-projections of confocal images from time-lapse sequences of one wild-type, one rack-1(RNAi), one rab-11(RNAi), and one zyg-9(RNAi) embryo from a GFP-DNC-2 strain are shown. (A-D) Wild-type embryo, also see Supplemental Movie 9. GFP-DNC-2 is enriched around the centrosomes (A) and on the spindle microtubules during metaphase (B) and anaphase (C). (E-H) rack-1 (RNAi) embryo, also see Supplemental Movie 10. RACK-1 depletion reduces the intensity of GFP-DNC-2 on the spindle (E and F). GFP-DNC-2 aggregates in cytoplasm (E-G). (I-L) rab-11(RNAi) embryo; also see Supplemental Movie 11. RAB-11 depletion leads to spindle alignment defect (J and K) but does not reduces the intensity of GFP-DNC-2 at centrosomes or on the spindle (I-K). There is no cytoplasmic aggregation of GFP-DNC-2. (M-P) zyg-9(RNAi) embryo. Sperm pronucleus forms spindle before oocyte pronucleus migration (N). GFP-DNC-2 localizes to the spindle (N-O). (Q) RACK-1 and DNC-2 interact in vivo. Whole worm lysates from GFP-DNC-2 worms were immunoprecipitated with anti-GFP antibody and immunoblotted with anti-RACK-1 antibody. WT worm lysate was used as a negative control (middle lane). Input is $1 / 100$ th of the IP. Numbers indicate molecular weight markers (kilodaltons). Bar, $10 \mu \mathrm{m}$.

served in DNC-2 depleted embryos (data not shown). We conclude that shorter astral microtubules do not contribute to the mislocalization of the RE and DNC-2 in rack-1(RNAi) embryos. Our data suggest that RACK-1 is required to target both RAB-11 endosomes and DNC-2 properly during the cell cycle.

\section{RACK-1 Directly Interacts with DNC-2 In Vivo}

RACK-1 was originally found to interact with DNC-2 in a yeast-two-hybrid study in C. elegans (Li et al., 2004). To validate whether RACK-1 and DNC-2 physically interact in vivo, we performed immunoprecipitation experiments using GFP-DNC-2 worm lysates. Using an antibody against GFP, we were able to identify RACK-1 in the precipitant (Figure 5Q). As a negative control, the same GFP antibody was unable to identify RACK-1 from wild-type (N2) worm lysates, indicating the RACK-1/DNC-2 interaction is dependent on the presence of GFP-DNC-2. These results are consistent with yeast two-hybrid studies (Li et al., 2004) and suggest that RACK-1 and DNC-2 physically interact in vivo. These data provide a biochemical basis for the functional relationship between these two proteins. RACK-1 likely binds to DNC-2 and is necessary to target the dynein/dynactin motor, which regulates the dynamics of recycling endosomes during mitosis.

\section{DISCUSSION}

In summary, our results show that RACK-1 is required for furrow invagination and abscission during cytokinesis. In the

Figure 6. RAB-11 and DNC-2 are not required for RACK-1 localization at centrosomes. Representative images are from wild-type (A-D), rab-11(RNAi) (E-H), and dnc-2(RNAi) (I-L) embryos stained with anti-RACK1 antibody. Prometaphase, metaphase, and anaphase are shown. Stages are determined by DAPI staining (data not shown). In wild type, RACK-1 localizes to centrosomes from pronuclear migration $(\mathrm{A}-\mathrm{C})$. During metaphase RACK-1 concentrates to centrosomes and faintly localizes to kinetochores region (B). In $r a b-11(R N A i)$ and dnc-2(RNAi) embryos RACK-1 also localizes to centrosomes (D-F and G-I). Bar, $10 \mu \mathrm{m}$.
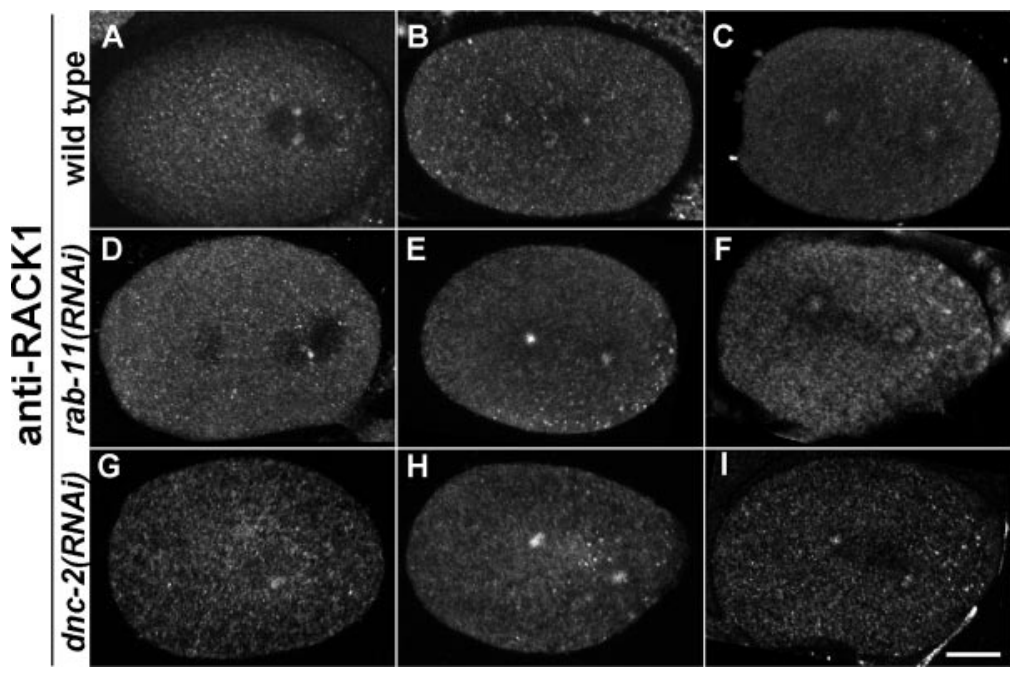
early embryo, RACK-1 is localized to centrosomes, kinetochores, nuclear envelopes, and midbodies. RACK-1 depletion leads to abnormal chromosome segregation, shorter astral microtubules, and disruption of germline membrane organization. We find that RACK-1 is required for the recruitment of RAB-11-labeled recycling endosomes to the pericentrosomal region and the spindle. Further studies indicate that RACK-1 directly binds to the C. elegans p50/dynamitin subunit of the dynactin complex, DNC-2. Collectively, our data suggest that RACK-1 is necessary for the cellular localization of recycling endosomes by interacting with dynactin. We believe we have identified a molecular cue that directs the proper targeting of dynactin and recycling endosomes during mitosis, ensuring proper membrane dynamics and targeting during both phases of cytokinesis.

\section{RACK-1 Regulates RE Distribution and Is Required for Cytokinesis}

Recycling endosomes have been shown to be a major source for membrane addition along the cleavage furrow and at the abscission site during cytokinesis (van Dam and Stoorvogel, 2002; Riggs et al., 2003). The RE is recruited to the pericentrosomal region before furrow initiation (Maxfield and McGraw, 2004). However, factors that target the RE during the cell cycle are unknown. In this work, we have identified RACK-1 as a significant factor required for the sequestration of recycling endosomes to the centrosomes and the spindle. We propose that RACK-1 facilitates the sequestration the recycling endosomes to the centrosomes via the p50/dynamtin subunit of the dynactin complex. To our knowledge, this work is the first to show the role of RACK-1 in regulating endomembrane dynamics during cytokinesis.

Why do rack-1(RNAi) embryos display cytokinesis defects? We have shown that RACK-1 is required for the recruitment of REs to the pericentrosomal region before the metaphase-anaphase transition. RACK-1 knockdown via RNAi led to a significant reduction of REs around the centrosomes during prometaphase and metaphase when compared with WT at this time. During anaphase, we believe the reduction of RE around centrosomes leads to a reduction of endosomal vesicles destined to be delivered to the ingressing cleavage furrow and midbody. The overall result is the failure to fully invaginate furrow membrane and complete abscission events.

Shorter astral microtubules and cytokinesis defects were rarely observed in $d n c-2(R N A i)$ embryos, however rack1(RNAi) embryos predominantly displayed these defects. So why is this the case? Data from other systems has hinted at a role for dynein and the dynactin complex in cytokinesis (Karki et al., 1998; Strickland et al., 2005; Delcros et al., 2006). Evidence in C. elegans has also shown that dynein heavy chain $(d h c-1$ ts $)$ mutants at the restrictive temperature lead to failures in cell division (Schmidt et al., 2005). Recent evidence has shown that the dynactin complex can target specific cytokinesis factors to the spindle midzone (Delcros et al., 2006); however, the exact role of the p50 subunit of the dynactin complex/DNC-2 in cytokinesis is unclear. It is possible that there are redundant mechanisms involved to ensure successful cytokinesis with respect to the dynein/ dynactin complex. Future experiments will elucidate these possibilities.

\section{RACK-1 Depletion Shares Similar Phenotypes with RAB-11 Depletion}

rack-1(RNAi) embryos are phenotypically similar to rab11(RNAi) embryos in several aspects. Depletion of RACK-1 and RAB-11 (Skop et al., 2001) both lead to cytokinesis failures. Polarbody extrusion defects, osmotic sensitivity, sterility, and shorter astral microtubules were also observed (Zhang et al., 2008b). These similarities suggest that RACK-1 and RAB-11 may be functioning in the same pathway, namely, by regulating recycling endosomes targeting and dynamics during mitosis. However, it is less likely that the only role of RACK-1 is to recruit the RE to centrosomes and the spindle, considering other well-studied roles of RACK1 in PKC signaling (Mochly-Rosen et al., 1995), cell migration (Cox et al., 2003), and translation (Nilsson et al., 2004). Our study indicates that RACK-1 localizes to cellular loci where RAB-11 does not (such as kinetochores and nuclear envelopes) and is involved in processes where RAB-11 is not (such as chromosome segregation). This pleiotropic nature of RACK-1 is not unexpected. Being a scaffolding protein, RACK-1 may function in tethering other complexes and may be required in multiple cellular events.

\section{RACK-1 Targets DNC-2 to Specific Cellular Foci}

To our knowledge, our data are the first to show a direct physical interaction of RACK-1 with DNC-2, the C. elegans p50/dynamitin subunit of the dynactin complex. RACK-1 has been shown to bind dynein light chain 1 (Zhang et al., 2008c), but not to the dynactin complex that we have presented here. We believe that RACK-1 may play a role in the regulation of the dynactin complex during development. We have shown that RACK-1 is necessary for DNC-2 localization to centrosomes and the spindle. These findings raise possibilities that RACK-1 may function during other cellular events to target dynactin complexes. rack-1(RNAi) embryos also display chromosome congression defects, consistent with the finding that eliminating kinetochore dynein in mammalian cells remarkably reduces congression efficiency (Li et al., 2007). RACK-1 might facilitate the proper localization of dynein/dynactin to the kinetochore where RACK-1 resides (Figure 1Q), thus promoting kinetochore microtubule capturing, but this has yet to be determined.

Interestingly, RACK-1 depletion also does not lead to spindle alignment defects, as seen in $d n c-2$ (RNAi) embryos (Skop and White, 1998). The localization of DNC-2 to the centrosomes and spindle is likely not necessary for spindle alignment. Dynein/dynactin complexes contribute to the generation of microtubule pulling forces, which are required for spindle alignment in the posterior of the embryo (Skop and White, 1998; Gonczy et al., 1999). Our results suggest that RACK-1 depletion may not affect cortical dynein/dynactin localization and activity, as well as force generation.

RACK-1 and DNC-2 may exhibit mutually dependent localization. We examined RACK-1 localization in $d n c-2$ (RNAi)-treated embryos and determined that knockdown of DNC-2 did not disrupt RACK-1 localization at centrosomes (Figure 5). These data suggest that DNC-2 may not be required for targeting RACK-1 to particular foci. However, because RNAi may not completely deplete DNC-2, we cannot rule out the possibility that a small amount of DNC-2 was sufficient to localize RACK-1. Future experiments will elucidate these possibilities.

\section{ACKNOWLEDGMENTS}

We thank Drs. Haining Zhang, John White, Anne Spang, Andy Golden, Karen Oegema, and the Caenorhabditis Genome Consortium for generously providing us strains and reagents. We are grateful to Drs. John White, Akihiro Ikeda, William Bement, and Koen Verbrugghe for critically reading the manuscript. We also want to thank Yuji Nakayama, Jessica Shivas, Mary Kate Bonner, and Thomas Dietz for comments and help. This work was supported by National Science Foundation CAREER award (MCB-0546398) (to A.R.S.). 


\section{REFERENCES}

Abramoff, M. D., Magelhaes, P. J., and Ram, S. J. (2004). Image processing with ImageJ. Biophotonics Int. 11, 36-42.

Albertson, R., Riggs, B., and Sullivan, W. (2005). Membrane traffic: a driving force in cytokinesis. Trends Cell Biol. 15, 92-101.

Audhya, A., Hyndman, F., McLeod, I. X., Maddox, A. S., Yates, J. R., 3rd, Desai, A., and Oegema, K. (2005). A complex containing the Sm protein CAR-1 and the RNA helicase CGH-1 is required for embryonic cytokinesis in Caenorhabditis elegans. J. Cell Biol. 171, 267-279.

Brenner, S. (1974). The genetics of Caenorhabditis elegans. Genetics 77, 71-94.

Chase, D., Serafinas, C., Ashcroft, N., Kosinski, M., Longo, D., Ferris, D. K., and Golden, A. (2000). The polo-like kinase PLK-1 is required for nuclear envelope breakdown and the completion of meiosis in Caenorhabditis elegans. Genesis 26, 26-41.

Cox, E. A., Bennin, D., Doan, A. T., O'Toole, T., and Huttenlocher, A. (2003). RACK1 regulates integrin-mediated adhesion, protrusion, and chemotactic cell migration via its Src-binding site. Mol. Biol. Cell 14, 658-669.

Danilchik, M. V., Bedrick, S. D., Brown, E. E., and Ray, K. (2003). Furrow microtubules and localized exocytosis in cleaving Xenopus laevis embryos. J. Cell Sci. 116, 273-283.

Delcros, J. G., Prigent, C., and Giet, R. (2006). Dynactin targets Pavarotti-KLP to the central spindle during anaphase and facilitates cytokinesis in Drosophila S2 cells. J. Cell Sci. 119, 4431-4441.

Dell, E. J., Connor, J., Chen, S., Stebbins, E. G., Skiba, N. P., Mochly-Rosen, D., and Hamm, H. E. (2002). The betagamma subunit of heterotrimeric G proteins interacts with RACK1 and two other WD repeat proteins. J. Biol. Chem. 277, $49888-49895$.

Emoto, K., and Umeda, M. (2000). An essential role for a membrane lipid in cytokinesis. Regulation of contractile ring disassembly by redistribution of phosphatidylethanolamine. J. Cell Biol. 149, 1215-1224.

Fielding, A. B., Schonteich, E., Matheson, J., Wilson, G., Yu, X., Hickson, G. R., Srivastava, S., Baldwin, S. A., Prekeris, R., and Gould, G. W. (2005). Rab11FIP3 and FIP4 interact with Arf6 and the exocyst to control membrane traffic in cytokinesis. EMBO J. 24, 3389-3399.

Glotzer, M. (2005). The molecular requirements for cytokinesis. Science 307, 1735-1739.

Gonczy, P., Pichler, S., Kirkham, M., and Hyman, A. A. (1999). Cytoplasmic dynein is required for distinct aspects of MTOC positioning, including centrosome separation, in the one cell stage Caenorhabditis elegans embryo. J. Cell Biol. 147, 135-150.

Goss, J. W., and Toomre, D. K. (2008). Both daughter cells traffic and exocytose membrane at the cleavage furrow during mammalian cytokinesis. J. Cell Biol. 181, 1047-1054.

Gromley, A., Yeaman, C., Rosa, J., Redick, S., Chen, C. T., Mirabelle, S., Guha, M., Sillibourne, J., and Doxsey, S. J. (2005). Centriolin anchoring of exocyst and SNARE complexes at the midbody is required for secretory-vesiclemediated abscission. Cell 123, 75-87.

Hickson, G. R., Matheson, J., Riggs, B., Maier, V. H., Fielding, A. B., Prekeris, R., Sullivan, W., Barr, F. A., and Gould, G. W. (2003). Arfophilins are dual Arf/Rab 11 binding proteins that regulate recycling endosome distribution and are related to Drosophila nuclear fallout. Mol. Biol. Cell 14, 2908-2920.

Kaitna, S., Mendoza, M., Jantsch-Plunger, V., and Glotzer, M. (2000). Incenp and an aurora-like kinase form a complex essential for chromosome segregation and efficient completion of cytokinesis. Curr. Biol. 10, 1172-1181.

Kamath, R. S., and Ahringer, J. (2003). Genome-wide RNAi screening in Caenorhabditis elegans. Methods 30, 313-321.

Karki, S., LaMonte, B., and Holzbaur, E. L. (1998). Characterization of the p22 subunit of dynactin reveals the localization of cytoplasmic dynein and dynactin to the midbody of dividing cells. J. Cell Biol. 142, 1023-1034.

King, S. J., Brown, C. L., Maier, K. C., Quintyne, N. J., and Schroer, T. A. (2003). Analysis of the dynein-dynactin interaction in vitro and in vivo. Mol. Biol. Cell 14, 5089-5097.

Kouranti, I., Sachse, M., Arouche, N., Goud, B., and Echard, A. (2006). Rab35 regulates an endocytic recycling pathway essential for the terminal steps of cytokinesis. Curr. Biol. 16, 1719-1725.

Lecuit, T., and Wieschaus, E. (2000). Polarized insertion of new membrane from a cytoplasmic reservoir during cleavage of the Drosophila embryo. J. Cell Biol. 150, 849-860.

Li, S., et al. (2004). A map of the interactome network of the metazoan C. elegans. Science 303, 540-543.
Li, Y., Yu, W., Liang, Y., and Zhu, X. (2007). Kinetochore dynein generates a poleward pulling force to facilitate congression and full chromosome alignment. Cell Res. 17, 701-712.

Matthews, L. R., Carter, P., Thierry-Mieg, D., and Kemphues, K. (1998) ZYG-9, a Caenorhabditis elegans protein required for microtubule organization and function, is a component of meiotic and mitotic spindle poles. J. Cell Biol. $141,1159-1168$

Maxfield, F. R., and McGraw, T. E. (2004). Endocytic recycling. Nat. Rev. Mol. Cell Biol. 5, 121-132.

McCahill, A., Warwicker, J., Bolger, G. B., Houslay, M. D., and Yarwood, S. J. (2002). The RACK1 scaffold protein: a dynamic cog in cell response mechanisms. Mol. Pharmacol. 62, 1261-1273.

Mishima, M., Kaitna, S., and Glotzer, M. (2002). Central spindle assembly and cytokinesis require a kinesin-like protein/RhoGAP complex with microtubule bundling activity. Dev. Cell 2, 41-54.

Mochly-Rosen, D., and Kauvar, L. M. (1998). Modulating protein kinase C signal transduction. Adv. Pharmacol. 44, 91-145.

Mochly-Rosen, D., Smith, B. L., Chen, C. H., Disatnik, M. H., and Ron, D. (1995). Interaction of protein kinase C with RACK1, a receptor for activated $\mathrm{C}$-kinase: a role in beta protein kinase $\mathrm{C}$ mediated signal transduction. Biochem. Soc. Trans. 23, 596-600.

Neer, E. J., Schmidt, C. J., Nambudripad, R., and Smith, T. F. (1994). The ancient regulatory-protein family of WD-repeat proteins. Nature 371, 297 300 .

Nilsson, J., Sengupta, J., Frank, J., and Nissen, P. (2004). Regulation of eukaryotic translation by the RACK1 protein: a platform for signalling molecules on the ribosome. EMBO Rep. 5, 1137-1141.

O'Connell, K. F., Leys, C. M., and White, J. G. (1998). A genetic screen for temperature-sensitive cell-division mutants of Caenorhabditis elegans. Genetics 149, 1303-1321.

Pelissier, A., Chauvin, J. P., and Lecuit, T. (2003). Trafficking through Rab11 endosomes is required for cellularization during Drosophila embryogenesis Curr. Biol. 13, 1848-1857.

Poteryaev, D., Fares, H., Bowerman, B., and Spang, A. (2007). Caenorhabditis elegans SAND-1 is essential for RAB-7 function in endosomal traffic. EMBO J. $26,301-312$

Praitis, V., Casey, E., Collar, D., and Austin, J. (2001). Creation of low-copy integrated transgenic lines in Caenorhabditis elegans. Genetics 157, 1217-1226.

Prekeris, R. (2003). Rabs, Rips, FIPs, and endocytic membrane traffic. Sci World J. 3, 870-880.

Riggs, B., Fasulo, B., Royou, A., Mische, S., Cao, J., Hays, T. S., and Sullivan, W. (2007). The concentration of Nuf, a Rab11 effector, at the microtubuleorganizing center is cell cycle regulated, dynein-dependent, and coincides with furrow formation. Mol. Biol. Cell 18, 3313-3322.

Riggs, B., Rothwell, W., Mische, S., Hickson, G. R., Matheson, J., Hays, T. S., Gould, G. W., and Sullivan, W. (2003). Actin cytoskeleton remodeling during early Drosophila furrow formation requires recycling endosomal components Nuclear-fallout and Rab11. J. Cell Biol. 163, 143-154.

Ron, D., Chen, C. H., Caldwell, J., Jamieson, L., Orr, E., and Mochly-Rosen, D. (1994). Cloning of an intracellular receptor for protein kinase C: a homolog of the beta subunit of G proteins. Proc. Natl. Acad. Sci. USA 91, 839-843.

Rothberg, K. G., Burdette, D. L., Pfannstiel, J., Jetton, N., Singh, R., and Ruben, L. (2006). The RACK1 homologue from Trypanosoma brucei is required for the onset and progression of cytokinesis. J. Biol. Chem. 281, 9781-9790.

Rothwell, W. F., Fogarty, P., Field, C. M., and Sullivan, W. (1998). Nuclearfallout, a Drosophila protein that cycles from the cytoplasm to the centrosomes, regulates cortical microfilament organization. Development 125, 1295-1303.

Rothwell, W. F., Zhang, C. X., Zelano, C., Hsieh, T. S., and Sullivan, W. (1999). The Drosophila centrosomal protein Nuf is required for recruiting Dah, a membrane associated protein, to furrows in the early embryo. J. Cell Sci. 112, 2885-2893.

Schmidt, D. J., Rose, D. J., Saxton, W. M., and Strome, S. (2005). Functional analysis of cytoplasmic dynein heavy chain in Caenorhabditis elegans with fast-acting temperature-sensitive mutations. Mol. Biol. Cell 16, 1200-1212.

Shuster, C. B., and Burgess, D. R. (2002). Targeted new membrane addition in the cleavage furrow is a late, separate event in cytokinesis. Proc. Natl. Acad. Sci. USA 99, 3633-3638.

Sisson, J. C., Field, C., Ventura, R., Royou, A., and Sullivan, W. (2000). Lava lamp, a novel peripheral Golgi protein, is required for Drosophila melanogaster cellularization. J. Cell Biol. 151, 905-918.

Sklan, E. H., Podoly, E., and Soreq, H. (2006). RACK1 has the nerve to act: structure meets function in the nervous system. Prog. Neurobiol. 78, 117-134. 
Skop, A. R., Bergmann, D., Mohler, W. A., and White, J. G. (2001). Completion of cytokinesis in C. elegans requires a brefeldin A-sensitive membrane accumulation at the cleavage furrow apex. Curr. Biol. 11, 735-746.

Skop, A. R., Liu, H., Yates, J., 3rd, Meyer, B. J., and Heald, R. (2004). Dissection of the mammalian midbody proteome reveals conserved cytokinesis mechanisms. Science 305, 61-66.

Skop, A. R., and White, J. G. (1998). The dynactin complex is required for cleavage plane specification in early Caenorhabditis elegans embryos. Curr. Biol. 8, 1110-1116.

Srayko, M., Quintin, S., Schwager, A., and Hyman, A. A. (2003). Caenorhabditis elegans TAC-1 and ZYG-9 form a complex that is essential for long astral and spindle microtubules. Curr. Biol. 13, 1506-1511.

Strickland, L. I., Wen, Y., Gundersen, G. G., and Burgess, D. R. (2005). Interaction between EB1 and p150glued is required for anaphase astral microtubule elongation and stimulation of cytokinesis. Curr. Biol. 15, 2249-2255.

Strome, S., Powers, J., Dunn, M., Reese, K., Malone, C. J., White, J., Seydoux, G., and Saxton, W. (2001). Spindle dynamics and the role of gamma-tubulin in early Caenorhabditis elegans embryos. Mol. Biol. Cell 12, 1751-1764.

Thompson, H. M., Skop, A. R., Euteneuer, U., Meyer, B. J., and McNiven, M. A. (2002). The large GTPase dynamin associates with the spindle midzone and is required for cytokinesis. Curr. Biol. 12, 2111-2117.

Timmons, L., Court, D. L., and Fire, A. (2001). Ingestion of bacterially expressed dsRNAs can produce specific and potent genetic interference in Caenorhabditis elegans. Gene 263, 103-112.
Ullrich, O., Reinsch, S., Urbe, S., Zerial, M., and Parton, R. G. (1996). Rab11 regulates recycling through the pericentriolar recycling endosome. J. Cell Biol. 135, 913-924.

Valetti, C., Wetzel, D. M., Schrader, M., Hasbani, M. J., Gill, S. R., Kreis, T. E., and Schroer, T. A. (1999). Role of dynactin in endocytic traffic: effects of dynamitin overexpression and colocalization with CLIP-170. Mol. Biol. Cell 10, 4107-4120.

van Dam, E. M., and Stoorvogel, W. (2002). Dynamin-dependent transferrin receptor recycling by endosome-derived clathrin-coated vesicles. Mol. Biol. Cell 13, 169-182.

Wilson, G. M., Fielding, A. B., Simon, G. C., Yu, X., Andrews, P. D., Hames, R. S., Frey, A. M., Peden, A. A., Gould, G. W., and Prekeris, R. (2005). The FIP3-Rab11 protein complex regulates recycling endosome targeting to the cleavage furrow during late cytokinesis. Mol. Biol. Cell 16, 849-860.

Won, M., Park, S. K., Hoe, K. L., Jang, Y. J., Chung, K. S., Kim, D. U., Kim, H. B., and Yoo, H. S. (2001). Rkp1/Cpc2, a fission yeast RACK1 homolog, is involved in actin cytoskeleton organization through protein kinase C, Pck2, signaling. Biochem. Biophys. Res. Commun. 282, 10-15.

Zhang, H., Skop, A. R., and White, J. G. (2008a). Src and Wnt signaling regulate dynactin accumulation to the P2-EMS cell border in C. elegans embryos. J. Cell Sci. 121, 155-161.

Zhang, H., Squirrell, J. M., and White, J. G. (2008b). RAB-11 permissively regulates spindle alignment by modulating metaphase microtubule dynamics in Caenorhabditis elegans early embryos. Mol. Biol. Cell 19, 2553-2565.

Zhang, W., Cheng, G. Z., Gong, J., Hermanto, U., Zong, C. S., Chan, J., Cheng, J. Q., and Wang, L. H. (2008). RACK1 and CIS mediate the degradation of BimEL in cancer cells. J. Biol. Chem. 283, 16416-16426. 